\title{
Erratum: October 2016 cover caption
}

Nat. Methods 13(10), (2016); published online 28 September 2016; corrected after print 14 November 2016

In the version of the cover caption initially published, the cover image was credited to Ruiyao Cai and Ali Ertürk with edits by Erin Dewalt. The description should have stated the cover image was created by Ruiyao Cai, Chenchen Pan, Francesca Paola Quacquarelli, Alireza Ghasemigharagoz and Ali Ertürk and edited by Erin Dewalt. The error has been corrected in the HTML and PDF versions of the caption. 